\title{
Paravertebral Block with Compound Betamethasone in Laparoscopic Cholecystectomy: A Double-blind Randomized Controlled Trial
}

\author{
Jinlei $\mathrm{Li}^{1}$, Lili Li ${ }^{2}$, Xiaoliang Zhang ${ }^{2}$, Cong Li ${ }^{2}$, Dong He ${ }^{2}$, Jian Zhang ${ }^{2}$, Chenxia Duan ${ }^{2}$, Francisco \\ Perese $^{3}$, Andrew Burzynski ${ }^{4}$, Christopher L. Wu ${ }^{5}$, Feng Dai ${ }^{6}$, Yun Xue ${ }^{7}$ \\ 1. Anesthesiology, Yale School of Medicine, New Haven, USA 2. Pain, Fuling Central Hospital of Chongqing City, \\ Chongqing, CHN 3. Anesthesiology, Somnia Anesthesia, Memorial Medical Center, Las Cruces, USA 4. Pain, Yale \\ University, New Haven, USA 5. Anesthesiology, Hospital for Special Surgery/Weill Cornell Medical College, New York, \\ USA 6. Biostatistics, Yale University, New Haven, USA 7. Anesthesiology, Fuling Central Hospital of Chongqing City, \\ Chongqing, $\mathrm{CHN}$
}

Corresponding author: Jinlei Li, jinlei.li@yale.edu

\section{Abstract \\ Backgrounds}

The aim of this study was to evaluate the utility of unilateral single injection thoracic paravertebral block (TPVB) with and without the addition of betamethasone for the acute pain management of patient's undergoing laparoscopic cholecystectomy (LC).

\section{Methods}

Eligible patients were allocated randomly to three groups: (A) general anesthesia followed by surgeon infiltration at port sites with ropivacaine $(n=48)$, (B) general anesthesia after single injection TPVB at right T7-8 level with ropivacaine only, Ropi_TPVB $(n=43)$, and $(C)$ general anesthesia after single injection TPVB with ropivacaine plus betamethasone, Ropi_Betamet_TPVB $(n=45)$. Primary outcome was TPVB duration assessed by the number of dermatomes at regular intervals up to 72 hours (h). Secondary outcomes included pain scores, analgesics consumption, and perioperative functional outcomes.

\section{Results}

The addition of betamethasone to ropivacaine in TPVB resulted in similar onset but significantly slower block regression between $4 \mathrm{~h}$ and $72 \mathrm{~h}$ as compared to ropivacaine alone $(\mathrm{P}<0.001)$. When compared to the surgeon infiltration group, Ropi_TPVB and Ropi_Betamet_TPVB group had significantly lower pain scores for $24 \mathrm{~h}$ and $48 \mathrm{~h}$, respectively, $\mathrm{P} \leqslant 0.001$. Both TPVB groups had less frequency of analgesics administration for $72 \mathrm{~h}, \mathrm{P}<0.001$, and earlier mobilization, $\mathrm{P}<0.001$.

Received 10/14/2019

Review began 10/25/2019 Review ended 10/25/2019 Published 10/29/2019

\section{() Copyright 2019}

$\mathrm{Li}$ et al. This is an open access article distributed under the terms of the Creative Commons Attribution License CC-BY 3.0., which permits unrestricted use, distribution, and reproduction in any medium, provided the original author and source are credited.

\section{Conclusions}

The addition of betamethasone to TPVB significantly prolonged block duration as compared to local anesthetic alone. TPVB both with and without the addition of betamethasone resulted in better perioperative analgesia and improved functional status when compared to surgical site local anesthetic infiltration.

Categories: Anesthesiology, Pain Management, General Surgery

Keywords: thoracic paravertebral, betamethasone, particulate glucocorticoid, laparoscopic cholecystectomy, block duration

\section{Introduction}

Laparoscopic cholecystectomy (LC) is a common and high-volume surgical procedure worldwide. It was estimated that approximately 5.5 million cases were performed in non-federal community hospitals in the United States alone between 1998 and 2006 [1]. While considered a relatively less invasive alternative compared to open cholecystectomy, LC nevertheless constitutes a profound insult to patients with the duration of pain and post-operative functional recovery ranging from one to seven days. Regional anesthetic interventions are at the center of a myriad of acute pain management maneuvers. In particular, thoracic paravertebral blocks (TPVB) have proven to be as effective as the more invasive epidural anesthesia in treating somatic/visceral pain and promoting positive recovery metrics in abdominal procedures [2].

In this randomized, double-blind, controlled study of LC patients, we specifically investigated the effects of unilateral TPVB with or without adjuvants, combination of non-particulate and particulate betamethasone, versus surgeon infiltration at port sites, on blockade duration, pain control and other patient-centered perioperative outcomes. The regression of sensory block, an important factor for analgesia duration, was 
well studied in epidural anesthesia [3, 4], but nearly none was documented in paravertebral block. Our hypotheses were: (1) Perineural administration of betamethasone together with local anesthetic would prolong the duration of TPVB blockade; and (2) TPVB would provide superior post-operative analgesia as compared to surgeon infiltration. To our knowledge, this is the first randomized controlled trial (RCT) that investigates the prolongation effects of a mixture of non-particulate and particulate glucocorticoids such as betamethasone on TPVBs in the operating room and immediately postoperatively.

Clinical Trial Registration: This study was registered at http://www.chictr.org.cn, identifier ChiCTR1800016696.

\section{Materials And Methods \\ Study design}

This was a single center, prospective, randomized, double-blind, and controlled study with an institutional approval from the Ethic Committee of Fuling Central Hospital of Chongqing City in China. During the study period between April 12, 2017 and April 12, 2018, 283 LC patients were identified, 117 were excluded, 16 declined, therefore 150 patients were enrolled. Inclusion criteria consisted of: (1) age 18 to 75 years; (2) American Society of Anesthesiologists (ASA) status I-II; (3) elective LC; (4) weight >40 kg; (5) ability to selfadminister opioid via PCA (standard of care in study hospital); and (6) overnight admission. Exclusion criteria included: (1) patient refusal, or patients with psychiatric or cognitive disorders; (2) a positive pregnancy test; (3) thoracic vertebral anomalies such as scoliosis or prior thoracic spine surgery; (4) coagulopathy; (5) systemic infection or local infection at planned injection sites; (6) allergy to any medications used in the study; (7) preexisting glucocorticoid use; (8) chronic pain; (9) diabetes mellitus (DM); (10) existing neuropathy. After an informed consent was obtained, 150 patients were divided into three groups per randomization table according to the pre-generalized random list in a 1:1:1 ratio: Local anesthetic Infiltration Analgesia Group (Control group), Ropivacaine single-injection TPVB Group (Ropi_TPVB), Ropivacaine \& Betamethasone single-injection TPVB Group (Ropi_Betamet_TPVB).

\section{Study protocol}

1. Intraoperative Study Protocol

Upon entering the operating room, patients were monitored with ASA standard monitors, including SpO2, ECG, and non-invasive blood pressure. After infusion of $10 \mathrm{ml} / \mathrm{kg}$ lactate ringer was initiated, patients were given intravenous midazolam $0.04 \mathrm{mg} / \mathrm{kg}$ and sufentanil $0.1 \mathrm{mcg} / \mathrm{kg}$.

All TPVBs were performed by the same investigator (LL), an experienced attending anesthesiologist. Under B mode-ultrasound guidance (Sonosite, S-Nerve), A 22-gauge Tuohy needle (B. Braun Medical Inc., Bethlehem, PA) was used to identify the right T7-8 paravertebral space under sterile condition in sitting position. A syringe filled with normal saline was connected to the needle. Upon identification of T7 transverse process, the needle was withdrawn slightly and re-directed caudally under the T7 transverse process. The needle was advanced until passing costotransverse ligament and $1 \mathrm{ml}$ of normal saline was injected into paravertebral space. After visualization of intervertebral space expansion and pleural displacement away from the ultrasound probe under direct visualization, the patients in group Ropi_TPVB or Ropi_Betamet_TPVB would be injected with $0.5 \%$ ropivacaine hydrochloride (AstraZeneca) $14 \mathrm{ml}+0.9 \%$ normal saline $1 \mathrm{ml}$, or $0.5 \%$ ropivacaine hydrochloride $14 \mathrm{ml}+2.5 \mathrm{mg}$ betamethasone dipropionate phosphate/1 mg betamethasone sodium phosphate in $1 \mathrm{ml}$ (Merck Sharp \& Dohme), respectively. The onset of block was assessed 15 minutes after block completion by evaluation of patient's sensation to discern temperature change (with ice).

For all groups, an intravenous (IV) general anesthesia induction was then performed with intravenous propofol, sufentanil and rocuronium followed by endotracheal intubation.

Before surgical incision, patients in the control group were infiltrated with $0.5 \%$ ropivacaine $15 \mathrm{ml}$ by the surgeon per standard protocols in this institute, with port I immediately to the right of the umbilicus, port II immediately below the xiphoid and $3 \mathrm{~cm}$ to the right of the midline, and port III $5 \mathrm{~cm}$ below the rib cage at right midclavicular line.

Anesthesia were subsequently maintained with a mixture of oxygen, air, and sevoflurane with end-tidal CO2 between 35 and $40 \mathrm{mmHg}$. Sufentanil $0.1 \mathrm{mcg} / \mathrm{kg}$, up to $5 \mathrm{mcg}$, was injected for any increase in arterial blood pressure and/or heart rate $>20 \%$ from baseline. All subjects received ondansetron $0.1 \mathrm{mg} / \mathrm{kg}$, up to $4 \mathrm{mg}$, at the end of surgery.

\section{Postoperative Study Protocol}

All subsequent follow-ups were performed by the same investigator (XZ). Analgesic block levels and duration were assessed using patient's temperature sensation change (with ice). The primary outcome was TPVB duration via the number of dermatomes affected on the ipsilateral side of TPVB placement, as a measure of 
duration of TPVB, assessed at $0.5 \mathrm{~h}, 2 \mathrm{~h}, 4 \mathrm{~h}, 8 \mathrm{~h}, 12 \mathrm{~h}, 24 \mathrm{~h}, 48 \mathrm{~h}$ and $72 \mathrm{~h}$ starting when patients left recovery room. Secondary outcomes included pain scores measured on a 0 to 10 numeric pain scale (NPS), the frequency and dosage of analgesics including oral and intravenous (IV) nonsteroidal anti-inflammatory agents (NSAIDs), and IV opioids up to $72 \mathrm{~h}$ at discharge. Surgical outcomes and quality metrics included preoperative and postoperative serum glucose level, first time ambulation, length of hospital stay, as well as any side effects such as dizziness, nausea, vomiting, drowsiness, hypotension, respiratory inhibition, intestinal paralysis/ileus, delayed wound healing, and wound infection were also measured. After hospital discharge, the subjects were followed up via phone calls at one week, one month, and three months evaluating for pain at surgical incision sites as well as TPVB injections sites using NPS, amount of analgesics usage, potential adverse events such as nausea, emesis, itching, and infection.

\section{Statistical Analysis}

Data are presented as mean (SD), or median (interquartile range (IQR): 25 th percentile - 75 th percentile) for continuous variables, and frequency and percentage for categorical variables. The analysis of our primary outcome, the number of dermatomes at different follow-up time points, was performed by fitting a linear mixed model with the use of SAS Proc Mixed procedure, in which fixed effects including group (Ropi_Betamet_TPVB vs. Ropi_TPVB), time $(0.25$ h, 0.5 h, 2 h, 4 h, 8 h, 12 h, 24 h, 48 h, 72 h), time by group interaction term were adjusted as covariates in the mean model. An autoregressive covariance matrix was specified to account for correlations of repeated observations from the same individuals. The least square mean estimates and 95\% confidence intervals for the outcome at different time points were calculated. The statistical comparisons of secondary outcomes were performed using the Wilcoxon rank sum test for continuous variables and Fisher's exact test for categorical variables.

All the statistical analyses were performed using the statistical software SAS v9.4 (Cary, NC). A two-side pvalue of less than 0.01 was considered to be statistically significant.

\section{Sample Size Considerations}

The sample size for this trial was determined for the primary outcome, TPVB duration measured by the number of dermatomes changes with time number of dermatomes changed over time, to assess the prolongation effects of betamethasone on TPVB. A sample size of 45 subjects per group (Ropi_Betamet_TPVB, Ropi_TPVB) was required to provide $80 \%$ power to detect a standardized Cohen's d effect size of $0.7 \mathrm{SD}$ between the two TPVB groups with a significance level (alpha) of 0.01 using a two-sided two-sample unequal-variance t-test. To account for $\sim 10 \%$ attrition rate due to loss of follow-up, we enrolled 50 subjects for both the Ropi_Betamet_TPVB and the Ropi_TPVB groups. An equal number of 50 subjects were enrolled for the control group, which would provide $80 \%$ power with an alpha level of 0.01 to detect Cohen's d of 0.7SD for a continuous secondary outcome using two-sample unequal-variance t-test or detect an effect size (Cohen's W) of 0.56 for a binary outcome using a 2 degrees of freedom Chi-Square test. Thus, in total, a sample size of $\mathrm{n}=150$ (50 per group) was selected.

\section{Results}

A flow chart illustrates the process of this study (Figure 1). 


\section{Cureus}

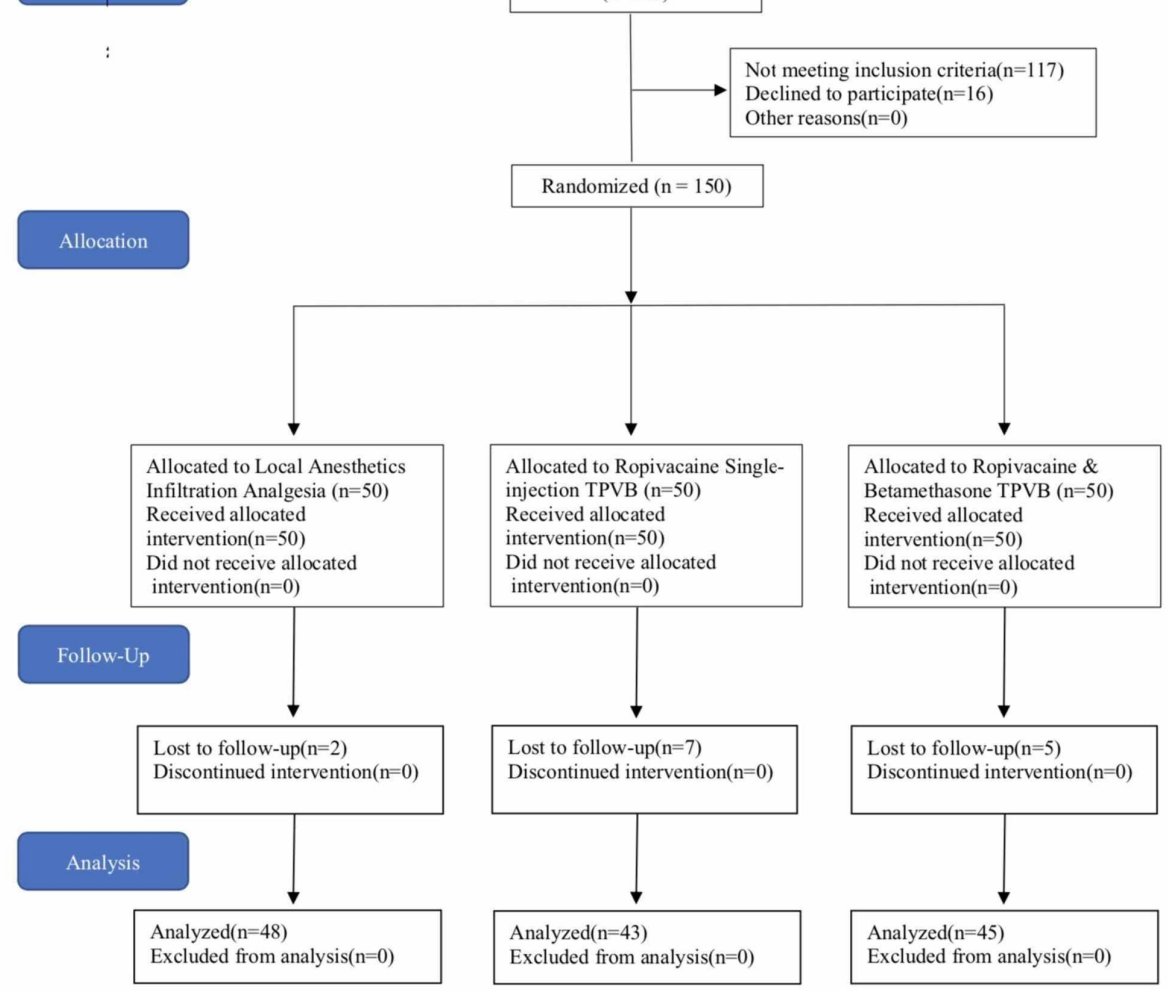

\section{FIGURE 1: Flow Diagram in CONSORT Format.}

TPVB: Thoracic paravertebral block

A total of 283 patients were assessed for eligibility, 117 were excluded, 16 declined to participate and 150 entered randomization. Originally 50 patients were assigned to each group, due to loss of follow-up after patients were discharged, a total of 136 patients completed this study. In the control group, 48 patients received local infiltration through randomization per protocol, in the Ropi_TPVB group, 43 patients received TPVB with plain ropivacaine, and in the Ropi_Betamet_TPVB group, 45 patients received TPVB with ropivacaine mixed with betamethasone. There was no statistically significant difference among the groups for age, gender, weight and ASA status (Table 1). 


\section{Cureus}

\begin{tabular}{|c|c|c|c|}
\hline Characteristics & Local infiltration $(\mathrm{N}=48)$ & Ropi_TPVB (N = 43) & Ropi_Betamet_TPVB $(\mathrm{N}=45)$ \\
\hline Age, yr & $48.19(12.63)$ & $51.79(11.40)$ & $47.98(14.47)$ \\
\hline Weight, kg & $61.40(9.47)$ & $62.00(10.23)$ & $60.67(12.00)$ \\
\hline \multicolumn{4}{|l|}{ Gender } \\
\hline Female & $26(054.17 \%)$ & $26(060.47 \%)$ & 28 (062.22\%) \\
\hline Male & $22(045.83 \%)$ & $17(039.53 \%)$ & $17(037.78 \%)$ \\
\hline \multicolumn{4}{|l|}{ ASA status } \\
\hline 1 & $34(070.83 \%)$ & $28(065.12 \%)$ & $29(064.44 \%)$ \\
\hline 2 & $14(029.17 \%)$ & $15(034.88 \%)$ & 16 (035.56\%) \\
\hline Serum glucose preop (mmol/L) & $5.13(0.52)$ & $5.17(0.50)$ & $5.07(0.42)$ \\
\hline
\end{tabular}

TABLE 1: Patient Demographics and Baseline Characteristics.

ASA: American Society of Anesthesiologists; TPVB: Thoracic paravertebral block.

\section{Primary outcome}

Betamethasone in TPVB resulted in longer blockade duration.

We chose LC patients for this study as LC has the highest volume in this study center, but postoperative opioid usage in this procedure is not high in general, therefore we used regression of dermatome as a surrogate marker for pain control and analgesic usage (Figure 2).

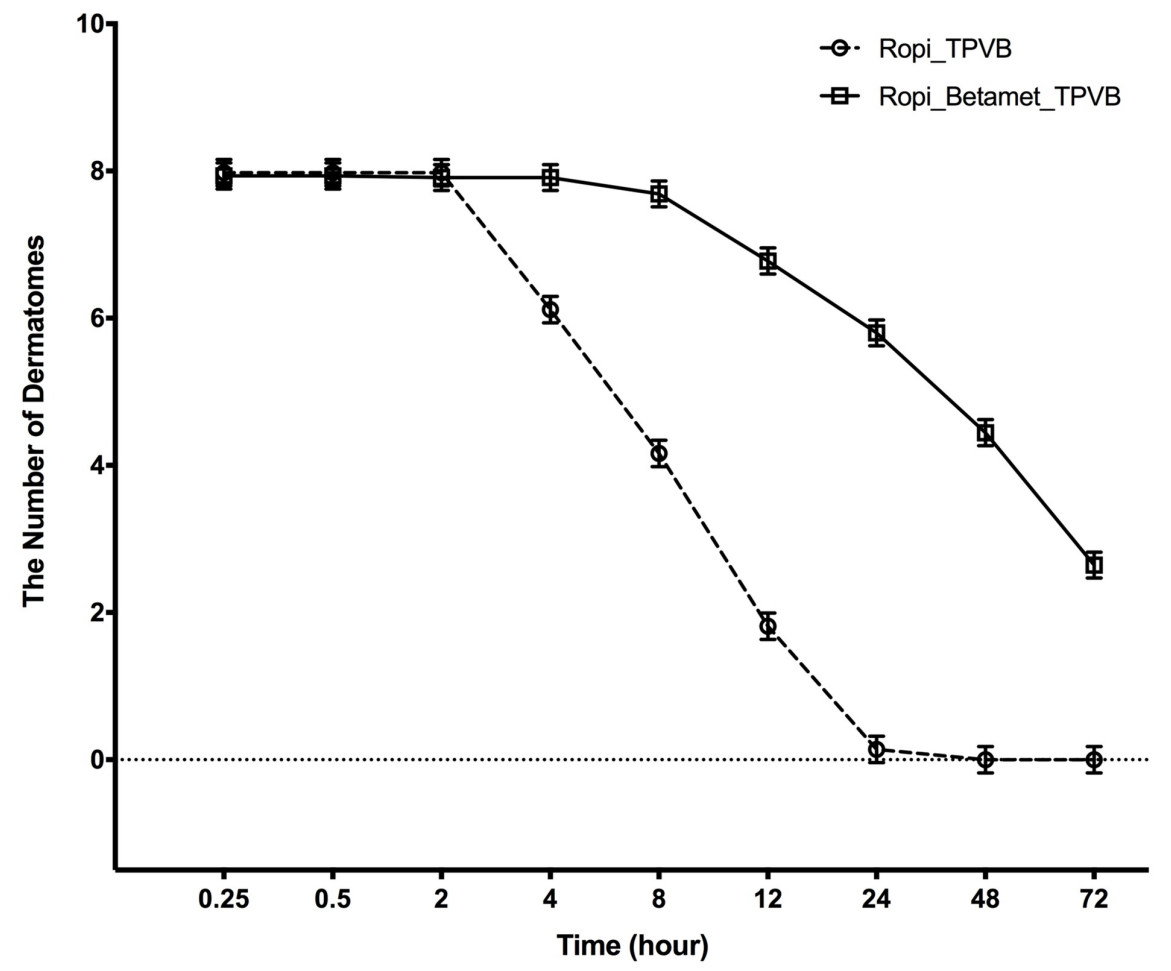

FIGURE 2: Chronological Changes in TPVB Dermatome Numbers.

TPVB: Thoracic paravertebral block

There was no significant difference on the block onset of TPVB with or without betamethasone as both 


\section{Cureus}

groups achieved approximately nearly eight levels of dermatomal coverage by $0.25 \mathrm{~h}$. Block duration assessment was initiated when patients left the recovery room. There was no significant difference among groups on the interval between block placement and the start of block duration assessment, which included the surgical duration and recovery room time. As shown in Table 2, the number of dermatomes was significantly higher in the Ropi_Betamet_TPVB group as compared to the Ropi_TPVB group between $4 \mathrm{~h}$ and $72 \mathrm{~h}(\mathrm{p}<0.001)$. At $24 \mathrm{~h}$, Ropi_TPVB patients had an average value of $0.14(0.09)$ for the dermatomal numbers of blockade, while the Ropi_Betamet_TPVB patients on average still had a value of 5.80 (0.09) (Table 2). The group difference of 5.66 (95\% CI: 5.38, 5.94) was statistically significant $(\mathrm{p}<0.001)$. By the time of 48 h, Ropi_TPVB patients had no residual blocks yet Ropi_Betamet_TPVB patients still had an average of 4.44 dermatomes of blockade (difference $=4.44$, 95\% CI: 4.19, 4.70, p < 0.001). Of note, starting at $4 \mathrm{~h}$ until $72 \mathrm{~h}$ post block placement, the dermatomal numbers being blocked between the two TPVB groups all indicated statistically significant differences $(\mathrm{P}<0.001)$.

\begin{tabular}{|c|c|c|c|c|}
\hline $\begin{array}{l}\text { Outcome: \# of dermatomes } \\
\text { at: }\end{array}$ & $\begin{array}{l}\text { Ropi_TPVB }{ }^{*} \text { (N = } \\
\text { 43) }\end{array}$ & $\begin{array}{l}\text { Ropi_Betamet_TPVB }{ }^{\star} \text { ( } \mathrm{N}= \\
\text { 45) }\end{array}$ & $\begin{array}{l}\text { Difference estimate }(95 \% \\
\text { Cl) }\end{array}$ & $\begin{array}{l}\mathrm{P}- \\
\text { value }\end{array}$ \\
\hline $0.25 \mathrm{~h}$ & $7.98(0.09)$ & $7.93(0.09)$ & $-0.04(-0.30,0.21)$ & 0.74 \\
\hline $0.5 \mathrm{~h}$ & $7.98(0.09)$ & $7.93(0.09)$ & $-0.04(-0.30,0.21)$ & 0.74 \\
\hline $2 \mathrm{~h}$ & $7.98(0.09)$ & $7.91(0.09)$ & $-0.07(-0.32,0.19)$ & 0.61 \\
\hline $4 \mathrm{~h}$ & $6.12(0.09)$ & $7.91(0.09)$ & $1.79(1.54,2.05)$ & $<0.001$ \\
\hline $8 \mathrm{~h}$ & $4.16(0.09)$ & $7.69(0.09)$ & $3.53(3.27,3.78)$ & $<0.001$ \\
\hline $12 \mathrm{~h}$ & $1.81(0.09)$ & $6.78(0.09)$ & $4.96(4.71,5.22)$ & $<0.001$ \\
\hline $24 \mathrm{~h}$ & $0.14(0.09)$ & $5.80(0.09)$ & $5.66(5.40,5.92)$ & $<0.001$ \\
\hline $48 \mathrm{~h}$ & $0(0.09)$ & $4.44(0.09)$ & $4.44(4.19,4.70)$ & $<0.001$ \\
\hline $72 \mathrm{~h}$ & $0(0.09)$ & 2.64 (0.09) & $2.64(2.39,2.90)$ & $<0.001$ \\
\hline
\end{tabular}

TABLE 2: Summary of TPVB Dermatome Numbers with Time.

*: Data are presented as least-square mean (standard error).

TPVB: Thoracic paravertebral block

\section{Secondary outcomes}

1. Postoperative pain scores in TPVB patients with or without betamethasone were lower as compared to surgical port site infiltration control patients.

Shown in Table 3, patients of the two TPVB groups had statistically lower pain scores than patients in surgical infiltration group at all times up to $24 \mathrm{~h}(\mathrm{p}<0.001)$. The Ropi_Betamet_TPVB group had significantly lower pain scores than the Ropi_TPVB group at time points $8 \mathrm{~h}$ up to discharge at $72 \mathrm{~h}$. The above differences in patient pain sensation were consistent with the TPVB dermatomal assessment shown in Figure 2, where there were significant differences of blockade effects between the Ropi_Betamet_TPVB group and Ropi_TPVB group. There was no incidence of persistent postsurgical pain in any group at one week, one month, and three months postoperatively. 


\section{Cureus}

\begin{tabular}{|c|c|c|c|c|c|c|}
\hline & & & & & P-value & \\
\hline Outcomes & $\begin{array}{l}\text { Control }(\mathrm{N}= \\
\text { 48) }\end{array}$ & $\begin{array}{l}\text { Ropi_TPVB (N = } \\
\text { 43) }\end{array}$ & $\begin{array}{l}\text { Ropi_Betamet_TPVB (N = } \\
\text { 45) }\end{array}$ & $\begin{array}{l}\text { Ropi_TPVB vs. } \\
\text { Control }\end{array}$ & $\begin{array}{l}\text { Ropi_Betamet_TPVB vs. } \\
\text { Control }\end{array}$ & $\begin{array}{l}\text { Ropi_Betamet_TPVB vs. } \\
\text { Ropi_TPVB }\end{array}$ \\
\hline Pain $0.5 \mathrm{~h}$ & $1.0(0.0-2.0)$ & $0.0(0.0-0.0)$ & $0.0(0.0-0.0)$ & $<0.001$ & $<0.001$ & 0.13 \\
\hline Pain $2 \mathrm{~h}$ & $2.0(1.5-3.0)$ & $0.0(0.0-0.0)$ & $0.0(0.0-0.0)$ & $<0.001$ & $<0.001$ & 0.06 \\
\hline Pain $4 \mathrm{~h}$ & $3.0(2.0-4.0)$ & $0.0(0.0-1.0)$ & $0.0(0.0-1.0)$ & $<0.001$ & $<0.001$ & 0.44 \\
\hline Pain $8 \mathrm{~h}$ & $3.0(2.0-3.0)$ & $2.0(1.0-2.0)$ & $0.0(0.0-1.0)$ & $<0.001$ & $<0.001$ & $<0.001$ \\
\hline Pain $12 \mathrm{~h}$ & $3.0(2.0-4.0)$ & $2.0(2.0-3.0)$ & $1.0(0.0-1.0)$ & $<0.001$ & $<0.001$ & $<0.001$ \\
\hline Pain $24 \mathrm{~h}$ & $2.0(1.0-2.0)$ & $1.0(1.0-2.0)$ & $0.0(0.0-1.0)$ & 0.001 & $<0.001$ & $<0.001$ \\
\hline Pain $48 \mathrm{~h}$ & $1.0(0.0-1.0)$ & $1.0(0.0-1.0)$ & $0.0(0.0-0.0)$ & 0.018 & $<0.001$ & 0.002 \\
\hline Pain $72 \mathrm{~h}$ & $0.0(0.0-0.5)$ & $0.0(0.0-1.0)$ & $0.0(0.0-0.0)$ & 0.43 & 0.041 & 0.006 \\
\hline Pain at 1 week & $0.0(0.0-0.0)$ & $0.0(0.0-0.0)$ & $0.0(0.0-0.0)$ & 1.00 & 1.00 & 1.00 \\
\hline Pain at 1 month & $0.0(0.0-0.0)$ & $0.0(0.0-0.0)$ & $0.0(0.0-0.0)$ & 1.00 & 1.00 & 1.00 \\
\hline Pain at 3 months & $0.0(0.0-0.0)$ & $0.0(0.0-0.0)$ & $0.0(0.0-0.0)$ & 1.00 & 1.00 & 1.00 \\
\hline Frequency of analgesics $72 \mathrm{~h}^{*}$ & $2.0(2.0-3.0)$ & $1.0(0.0-1.0)$ & $0.0(0.0-0.0)$ & $<0.001$ & $<0.001$ & $<0.001$ \\
\hline Tramodol_mg_IV & $0.0(0.0-50.0)$ & $0.0(0.0-0.0)$ & $0.0(0.0-0.0)$ & 0.05 & $<0.001$ & 0.15 \\
\hline Parecoxib_mg_IV & $80.0(80.0-80.0)$ & $0.0(0.0-40.0)$ & $0.0(0.0-0.0)$ & $<0.001$ & $<0.001$ & 0.008 \\
\hline Imrecoxib_mg_PO & $0.0(0.0-0.0)$ & $0.0(0.0-100.0)$ & $0.0(0.0-0.0)$ & 0.08 & 0.034 & $<0.001$ \\
\hline Dizziness, Yes & $12(025.00 \%)$ & $05(011.63 \%)$ & $00(000.00 \%)$ & 0.10 & $<0.001$ & 0.03 \\
\hline \multicolumn{7}{|l|}{ Nausea/Nomiting } \\
\hline 0 & $39(081.25 \%)$ & $37(086.05 \%)$ & $41(091.11 \%)$ & 1.00 & 0.53 & 0.42 \\
\hline $1-3$ & $09(18.75 \%)$ & $06(13.95 \%)$ & $04(8.89 \%)$ & & & \\
\hline Drowsiness, Yes & $00(000.00 \%)$ & $00(000.00 \%)$ & $00(000.00 \%)$ & 1 & 1.00 & 1.00 \\
\hline Hypotension, Yes & $00(000.00 \%)$ & $01(002.33 \%)$ & $00(000.00 \%)$ & 0.47 & 1.00 & 0.49 \\
\hline Respiratory depression, Yes & $00(000.00 \%)$ & $00(000.00 \%)$ & $00(000.00 \%)$ & 1 & 1.00 & 1.00 \\
\hline lleus, Yes & $00(000.00 \%)$ & $00(000.00 \%)$ & $00(000.00 \%)$ & 1 & 1.00 & 1.00 \\
\hline $\begin{array}{l}\text { Postop serum glucose }(\mathrm{mmo} / \mathrm{L}) \\
\text { at } 0.5 \mathrm{~h}\end{array}$ & $7.0(6.5-7.8)$ & $8(6.2-7.9)$ & $66(6.0-7.3)$ & 0.73 & 0.08 & 0.15 \\
\hline Delayed wound healing, Yes & $00(000.00 \%)$ & $01(002.33 \%)$ & $00(000.00 \%)$ & 0.47 & 1.00 & 0.49 \\
\hline Wound infection, Yes & $00(000.00 \%)$ & $00(000.00 \%)$ & $00(000.00 \%)$ & 1 & 1.00 & 1.00 \\
\hline First ambulation time, $\mathrm{h}$ & $6.0(3.0-8.0)$ & $2.0(1.0-2.0)$ & $2.0(1.0-2.0)$ & $<0.001$ & $<0.001$ & 0.88 \\
\hline Length of stay, day & $3.0(3.0-3.0)$ & $3.0(3.0-3.0)$ & $3.0(3.0-3.0)$ & 0.63 & 0.61 & 0.99 \\
\hline
\end{tabular}

\section{TABLE 3: Secondary Outcomes and Adverse Events.}

Data are presented as median (IQR) or $\mathrm{n}(\%)$. P-value from Wilcoxon rank sum test or Fisher's exact test was presented.

*: Frequency of analgesics $72 \mathrm{~h}$ : each time a rescue analgesic was given, it was counted as once.

2. Postoperative analgesics consumption in patients with TPVB as compared to patients with surgical port site infiltration.

Shown in Table 3, the frequency of analgesics requested/administrated was significantly higher in the 
surgical infiltration group (control) as compared to both Ropi_TPVB group and Ropi_Betamet_TPVB group (P $<0.001$ ). The administration frequency of supplemental analgesics in Ropi_Betamet_TPVB group was also significantly lower than that of the Ropi_TPVB group $(\mathrm{P}<0.001)$. The intravenous opioid Tramadol consumption in the Ropi_Betamet_TPVB group was significantly different than that in the control group $(\mathrm{P}<$ 0.001). However, no significant difference was found between the Ropi_TPVB group and the control group, and between the Ropi_TPVB group and the Ropi_Betamet_TPVB group, respectively. The amount of IV Parecoxib in both TPVB groups was statistically lower than control, $\mathrm{P}<0.001$. In addition, the usage of IV Parecoxib in the Ropi_Betamet_TPVB group was statistically lower than that of Ropi_TPVB group, $\mathrm{P}=0.008$.

3. Perioperative functional outcomes and adverse events between surgical infiltration and TPVB groups.

Postoperatively, the median (IQR) time of each patient's first-time ambulation in the control group, surgical infiltration only, was $6.0(3.0-8.0) \mathrm{h}$, which was significantly greater $(\mathrm{p}<0.001)$ than those in Ropi_Betamet_TPVB $(2(1.0-2.0) \mathrm{h})$, and Ropi_TPVB $(2(1.0-2.0) \mathrm{h})$ (Table 3). No dizziness was reported in the Ropi_Betamet_TPVB group, while the rates of it in the Ropi_TPVB and the control group were $11.63 \%$ and $25 \%$, respectively. No significant difference in nausea/vomiting was identified among three groups, likely due to the usage of both intravenous and oral NSAIDs prior to opioids. There was one isolated delayed wound healing, and one reported hypotension in the Ropi_TPVB group. There was no incidence of wound infection, drowsiness, respiratory depression or ileus in any group.

\section{Discussion}

Although there are several studies on the regression of spinal and epidural anesthesia, there are limited, if any, studies in TPVB in the current literature [3,5]. To the best of our knowledge, this is the first RCT to systemically evaluate the waning course of sensory TPVB. Our study of dermatomes using cold sensation demonstrated the regressions of A $\boldsymbol{\nabla}$ fiber blockade after TPVB, which correlated roughly with the trend of somatic and visceral analgesia diminishing.

The salutary effects and safety of glucocorticoids in analgesia are well established [6-9]. However, controversy continues as to if perineural glucocorticoids have similar or better analgesic effects when compared to intravenous glucocorticoids. While some studies showed no difference [10,11], others suggested perineural glucocorticoids have lower minimum dose and/or provide more effective analgesia at same dose than its intravenous counterpart [7, 12]. Hydrophilic/non-particulate glucocorticoid dexamethasone is most commonly used with the prolongation effect on peripheral nerve blockade for 6-8 $\mathrm{h}$ [13]. The search for longer acting local anesthetics or local anesthetic adjuvants with longer duration continues. Lipophilic/particulate glucocorticoids exhibit similar effectiveness and safety outside of intrathecal space as non-particulate counterparts $[9,14,15]$ but with longer duration $[16,17]$. Thus far particulate glucocorticoids in peripheral nerve blocks were only described in isolated studies [18-20]. The safety data for perineural glucocorticoids is lacking in general. Nonetheless, reported adverse events for particulate glucocorticoids were mostly related to intrathecal injection or intravascular injection, and no complications have been reported for peripheral nerve blocks [18, 20-23].

We chose to use betamethasone sodium phosphate/dipropionate combination for several reasons [1]. Betamethasone lacks mineral-corticoid activities and is of relatively smaller molecular size as compared to other glucocorticoids [2]. This combination of betamethasone is FDA approved, albeit for other indications than peripheral nerve block [3]. This combination of betamethasone has been used successfully in other peripheral nerve blocks such as great occipital nerve block [24], which was endorsed by the American Headache Society $[4,22]$. This combination of betamethasone together with ropivacaine has been used safely in brachial plexus interscalene block $[5,20]$. When betamethasone dipropionate was applied directly to the transected tibial nerve in a rat model, it promoted nerve regeneration rather than induced nerve injury, which is a common concern for perineural adjuvant administration [6,25]. Pharmacokinetics studies of intramuscular injection betamethasone phosphate/betamethasone dipropionate showed that the active metabolite of betamethasone dipropionate has a half-life of approximately three days [26]. To minimize systemic effects, we used half of the above intramuscular dose of betamethasone through the route of perineural administration.

When it comes to glucocorticoid activity, we used in this study the dose of $2.5 \mathrm{mg}$ of slow release betamethasone dipropionate phosphate and $1 \mathrm{mg}$ immediate action betamethasone sodium phosphate, which adds up to about $3.125 \mathrm{mg}$ dexamethasone in slow release format and $1.42 \mathrm{mg}$ of dexamethasone in immediate release format, with a total activity of approximately $4.5 \mathrm{mg}$ of dexamethasone, a common and arguably the optimal perineural dosing for dexamethasone as a local anesthetic adjuvant. Consistent with the small dose of betamethasone adopted in this study, we did not find any evidence of hyperglycemia, delayed wound healing or wound infection. We did not monitor serum glucose beyond the routine as recent multi-analysis of randomized control studies has shown a single dose perioperative dexamethasone has negligible effect on hyperglycemia, delayed wound healing or wound infection in non-diabetic and diabetic patients alike. Nonetheless, the lack of difference in serum glucose levels and wound infections is likely underpowered in the present study, future research may be directed to achieve the lowest possible dose of glucocorticoid until desired endpoints are met. Our study excluded diabetic patients, and it would be an interesting study when various types of diabetes, such as DM I, poorly 
controlled DM II, and well-controlled DM II, are involved.

In our study, the ultrasound-guided TPVB spread was approximately eight dermatomes by cold sensation, close to what was obtained by Cheema et al. [27], and was wider than what Cheema or Boezaart reported with radio-opaque dye or methylene blue, respectively [28, 29]. This could be due to the assessment technique (direct visualization of dye spread vs. cold sensation), block technique (ultrasound vs. landmark), and the relatively low BMI in our study patient.

One limitation of this study is blinding. The performance of TPVB is not without any risks and we did not feel it ethically justified to perform sham TPVB in control patients, therefore patients are not blinded in terms of if they received TPVB. Secondly, the assessment of block onset was not blinded, as the single investigator (LL) who prepared all the medications, performed all the TPVB procedures, also performed the very first TPVB block level assessment at $0.25 \mathrm{~h}$. However, all subsequent assessments were exclusively performed by a single blinded practitioner (XZ) after patients had left the recovery room. Another limitation of this study was the choice of LC procedure, which is common, has documented risk of persistent postsurgical pain, but typically not associated with a long length of hospital stay or significant disease burden. As a result, the assessments for TPVB duration were prematurely stopped at the time of discharge at $72 \mathrm{~h}$. In addition, other than the duration of blockade, pain level assessment, and analgesics consumption, some important outcome measures such as patient satisfaction and quality of recovery were not sought. Future studies of TPVB with particulate glucocorticoids in more invasive procedures with longer than $72 \mathrm{~h}$ of stay are warranted. Even though the TPVB had significant impacts on perioperative pain scores, analgesics consumption, and functional status, there were no changes on the length of stays in this study, as the surgeons were not comfortable to send any LC patients home earlier than routine. As a result, the investigators did not feel the need to include cost of hospitalization parameter in this study.

A major strength of this study was its in-depth review of dermatomal subsiding pattern over time. We believe this to be a unique aspect to this study not previously published. Our research suggested that paravertebral administration of local anesthetic and low dose non-particulate and particulate glucocorticoids can attain substantial effects on postoperative analgesia on LC patients without significant side effects or complications. This study serves, in part, as a proof of concept for TPVBs as a reliable intervention for post-operative analgesia in abdominal surgery. It is the first RCT that demonstrated the superiority of unilateral TPVB over surgical local anesthetic infiltration for LC, also the first RCT that investigated the effectiveness and safety of particulate glucocorticoid adjuvants in TPVB for acute perioperative pain management.

\section{Conclusions}

Our study is among the few that reported much longer than $24 \mathrm{~h}$ duration of single injection TPVB blockade achieved with a local anesthetic adjuvant resulting in improved perioperative analgesia and functional outcomes, and without significant adverse events.

\section{Additional Information}

\section{Disclosures}

Human subjects: Consent was obtained by all participants in this study. Ethic Committee of Fuling Central Hospital of Chongqing City in China issued approval 2017014. This was a single center, prospective, randomized, double-blind, and controlled study with an institutional approval from the Ethic Committee of Fuling Central Hospital of Chongqing City in China. Animal subjects: All authors have confirmed that this study did not involve animal subjects or tissue. Conflicts of interest: In compliance with the ICMJE uniform disclosure form, all authors declare the following: Payment/services info: All authors have declared that no financial support was received from any organization for the submitted work. Financial relationships: All authors have declared that they have no financial relationships at present or within the previous three years with any organizations that might have an interest in the submitted work. Other relationships: All authors have declared that there are no other relationships or activities that could appear to have influenced the submitted work.

\section{References}

1. Murphy MM, Ng SC, Simons JP, Csikesz NG, Shah SA, Tseng JF: Predictors of major complications after laparoscopic cholecystectomy: surgeon, hospital, or patient?. J Am Coll Surg. 2010, 211:73-80. 10.1016/j.jamcollsurg.2010.02.050

2. Kahokehr A, Sammour T, Zargar-Shoshtari K, Srinivasa S, Hill AG: Recovery after open and laparoscopic right hemicolectomy: a comparison. J Surg Res. 2010, 162:11-16. 10.1016/j.jss.2010.02.008

3. Kanai A, Osawa S, Suzuki A, Ozawa A, Okamoto H, Hoka S: Regression of sensory and motor blockade, and analgesia during continuous epidural infusion of ropivacaine and fentanyl in comparison with other local anesthetics. Pain Med. 2007, 8:546-553. 10.1111/j.1526-4637.2006.00174.x

4. Park EY, Kil HK, Park WS, Lee NH, Hong JY: Effect of epidural saline washout on regression of sensory and motor block after epidural anaesthesia with $2 \%$ lidocaine and fentanyl in elderly patients. Anaesthesia. 2009, 64:273-276. 10.1111/j.1365-2044.2008.05746.x 
5. Stienstra R, Gielen M, van Poorten F, Kroon JW: Spinal anesthesia with plain bupivacaine 0.5\%: regression of sensory and motor blockade with different temperatures of the anesthetic solution. Anesth Analg. 1989, 69:593-597.

6. Rijsdijk M, van Wijck AJ, Kalkman CJ, Yaksh TL: The effects of glucocorticoids on neuropathic pain: a review with emphasis on intrathecal methylprednisolone acetate delivery. Anesth Analg. 2014, 118:1097-1112. 10.1213/ANE.0000000000000161

7. Zorrilla-Vaca A, Li J: Dexamethasone injected perineurally is more effective than administered intravenously for peripheral nerve blocks: a meta-analysis of randomized controlled trials. Clin J Pain. 2018, 34:276-284. 10.1097/AJP.0000000000000519

8. Murphy GS, Szokol JW, Greenberg SB, Avram MJ, Vender JS, Nisman M, Vaughn J: Preoperative dexamethasone enhances quality of recovery after laparoscopic cholecystectomy: effect on in-hospital and postdischarge recovery outcomes. Anesthesiology. 2011, 114:882-890. 10.1097/ALN.0b013e3181ec642e

9. Cicala RS, Turner R, Moran E, Henley R, Wong R, Evans J: Methylprednisolone acetate does not cause inflammatory changes in the epidural space. Anesthesiology. 1990, 72:556-558. 10.1097/00000542199003000-00026

10. Abdallah FW, Johnson J, Chan V, et al.: Intravenous dexamethasone and perineural dexamethasone similarly prolong the duration of analgesia after supraclavicular brachial plexus block: a randomized, triplearm, double-blind, placebo-controlled trial. Reg Anesth Pain Med. 2015, 40:125-132. 10.1097/AAP.0000000000000210

11. Rosenfeld DM, Ivancic MG, Hattrup SJ, et al.: Perineural versus intravenous dexamethasone as adjuncts to local anaesthetic brachial plexus block for shoulder surgery. Anaesthesia. 2016, 71:380-388. 10.1111/anae.13409

12. Chun EH, Kim YJ, Woo JH: Which is your choice for prolonging the analgesic duration of single-shot interscalene brachial blocks for arthroscopic shoulder surgery? Intravenous dexamethasone $5 \mathrm{mg}$ vs. perineural dexamethasone $5 \mathrm{mg}$ randomized, controlled, clinical trial. Medicine (United States). 2016, 95:e3828. 10.1097/MD.0000000000003828

13. Kirkham KR, Jacot-Guillarmod A, Albrecht E: Optimal dose of perineural dexamethasone to prolong analgesia after brachial plexus blockade: a systematic review and meta-analysis. Anesth Analg. 2018, 126:270-279. 10.1213/ANE.0000000000002488

14. Zufferey P, Bulliard C, Gremion G, Saugy M, So A: Systemic effects of epidural methylprednisolone injection on glucose tolerance in diabetic patients. BMC Res Notes. 2011, 4:552. 10.1186/1756-0500-4-552

15. Habib G, Jabbour A, Artul S, Hakim G: Intra-articular methylprednisolone acetate injection at the knee joint and the hypothalamic-pituitary-adrenal axis: a randomized controlled study. Clin Rheumatol. 2014, 33:99103. 10.1007/s10067-013-2374-4

16. Kim D, Brown J: Efficacy and safety of lumbar epidural dexamethasone versus methylprednisolone in the treatment of lumbar radiculopathy: a comparison of soluble versus particulate steroids. Clin J Pain. 2011, 27:518-522. 10.1097/AJP.0b013e31820c53e0

17. Noe CE, Haynsworth RF Jr: Comparison of epidural Depo-Medrol vs. aqueous betamethasone in patients with low back pain. Pain Pract. 2003, 3:222-225. 10.1046/j.1533-2500.2003.03026.x

18. Stan T, Goodman EJ, Bravo-Fernandez C, Holbrook CR: Adding methylprednisolone to local anesthetic increases the duration of axillary block. Reg Anesth Pain Med. 2004, 29:380-381.

19. Cok OY, Eker HE, Akin S, Aribogan A: Paravertebral block with methylprednisolone for management of chronic pain after cholecystectomy. Reg Anesth Pain Med. 2011, 2:193.

20. Watanabe K, Tokumine J, Yorozu T, Moriyama K, Sakamoto H, Inoue T: Particulate-steroid betamethasone added to ropivacaine in interscalene brachial plexus block for arthroscopic rotator cuff repair improves postoperative analgesia. BMC Anesthesiol. 2016, 16:84. 10.1186/s12871-016-0251-9

21. Benzon HT, Chew TL, McCarthy RJ, Benzon HA, Walega DR: Comparison of the particle sizes of different steroids and the effect of dilution: a review of the relative neurotoxicities of the steroids. Anesthesiology. 2007, 106:331-338.

22. Blumenfeld A, Ashkenazi A, Napchan U, et al.: Expert consensus recommendations for the performance of peripheral nerve blocks for headaches--a narrative review. Headache. 2013, 53:437-446. 10.1111/head.12053

23. Eker HE, Cok OY, Aribogan A, Arslan G: Management of neuropathic pain with methylprednisolone at the site of nerve injury. Pain Med. 2012, 13:443-451. 10.1111/j.1526-4637.2011.01323.x

24. Ambrosini A, Vandenheede M, Rossi P, Aloj F, Sauli E, Pierelli F, Schoenen J: Suboccipital injection with a mixture of rapid- and long-acting steroids in cluster headache: a double-blind placebo-controlled study. Pain. 2005, 118:92-96. 10.1016/j.pain.2005.07.015

25. Yao P, Li P, Jiang JJ, Li HY: Anastomotic stoma coated with chitosan film as a betamethasone dipropionate carrier for peripheral nerve regeneration. Neural Regen Res. 2018, 13:309-316. 10.4103/1673-5374.226401

26. He C, Fan H, Tan J, Zou J, Zhu Y, Yang K, Hu Q: Pharmacokinetics of betamethasone and betamethasone 17-monopropionate in Chinese healthy volunteers after intramuscular injection of betamethasone phosphate/betamethasone dipropionate. Arzneimittelforschung. 2011, 61:417-420. 10.1055/s-0031-1296220

27. Cheema SP, Ilsley D, Richardson J, Sabanathan S: A thermographic study of paravertebral analgesia . Anaesthesia. 1995, 50:118-121. 10.1111/j.1365-2044.1995.tb15092.x

28. Cheema S, Richardson J, McGurgan P: Factors affecting the spread of bupivacaine in the adult thoracic paravertebral space. Anaesthesia. 2003, 58:684-687. 10.1046/j.1365-2044.2003.03189_1.x

29. Boezaart AP, Raw RM: Continuous thoracic paravertebral block for major breast surgery. Reg Anesth Pain Med. 2006, 31:470-476. 10.1016/j.rapm.2006.03.009 